\title{
Desenvolvimento do conceito de um equipamento para o tratamento de efluente de lavagem de carros por eletrólise
}

\author{
Development of the concept of an equipment for the treatment of car wash effluent by electrolysis \\ Desarrollo del concepto de un equipo para el tratamiento de efluentes de lavado de autos por \\ electrólisis
}

Recebido: 25/01/2022 | Revisado: 29/01/2022 | Aceito: 01/02/2022 | Publicado: 03/02/2022

Raul Schenkel

ORCID: https://orcid.org/0000-0002-5289-5374 Universidade de Passo Fundo, Brasil E-mail: 102771@upf.br

Márcio Walber

ORCID: https://orcid.org/0000-0002-4472-0937 Universidade de Passo Fundo, Brasil E-mail: mwalber@upf.br

Camila Favretto de Souza

ORCID: https://orcid.org/0000-0002-3919-4370 Universidade Feevale, Brasil E-mail: camila.favretto@hotmail.com Marcelo Hemkemeier

ORCID: https://orcid.org/0000-0001-6541-4827 Universidade de Passo Fundo, Brasil E-mail: marceloh@upf.br

\begin{abstract}
Resumo
O desperdício de recursos hídricos e o aumento do consumo de água em sistemas urbanos, tem gerado grandes problemas de escassez de água. O processo de lavagem de veículos é um dos grandes consumidores de água e também gera um enorme volume de efluentes potencialmente poluidores, sendo lançados diretamente ou com tratamentos inadequados à rede de esgoto e/ou pluvial. Este artigo teve como objetivo o desenvolvimento de um conceito de um equipamento para o tratamento de efluentes por eletrólise de uma estação de lavagem de veículos capaz de diminuir o impacto ambiental desta atividade através do tratamento e reuso do efluente. Para elaboração do conceito, foi utilizada a metodologia de desenvolvimento de produtos dos autores Pahl et al., especificamente as fases de planejamento e concepção, onde foram estabelecidos os requisitos de projeto e o conceito para o equipamento de tratamento de efluentes por eletrólise. O equipamento concebido foi composto por câmara eletrolítica com raspador de escuma seguido de decantador, além dos demais controles operacionais. A alimentação e descarga foi realizada por bombas centrífugas.
\end{abstract}

Palavras-chave: Recurso hídrico; Tratamento de efluentes; Tratamento eletrolítico; Impacto ambiental; Reuso.

\begin{abstract}
The waste of water resources and the increase in water consumption in urban systems, has generated major problems of water scarcity. The vehicle washing process is a major consumer of water and also generates an enormous volume of potentially polluting effluents, being released directly or with inadequate treatments to the sewage and / or pluvial network. This article aimed to develop a concept for an equipment for the treatment of effluents by electrolysis of a vehicle washing station capable of reducing the environmental impact of this activity through the treatment and reuse of the effluent. To elaborate the concept, the product development methodology of the authors Pahl et al., was used, specifically the planning and conception phases, where the project requirements and the concept for the equipment for the treatment of effluents by electrolysis were established. The designed equipment consisted of an electrolytic chamber with a foam scraper followed by a decanter, in addition to the other operational controls. Feeding and unloading was carried out by centrifugal pumps.
\end{abstract}

Keywords: Water resource; Wastewater treatment; Electrolytic treatment; Environmental impact; Reuse.

\section{Resumen}

El derroche de recursos hídricos y el aumento del consumo de agua en los sistemas urbanos, ha generado importantes problemas de escasez de agua. El proceso de lavado de vehículos es un gran consumidor de agua y además genera un enorme volumen de efluentes potencialmente contaminantes, siendo vertidos directamente o con tratamientos inadecuados a la red de alcantarillado y / o pluvial. Este artículo tuvo como objetivo desarrollar un concepto de equipo 
para el tratamiento de efluentes por electrólisis de una estación de lavado de vehículos capaz de reducir el impacto ambiental de esta actividad mediante el tratamiento y reutilización del efluente. Para la elaboración del concepto se utilizó la metodología de desarrollo de producto de los autores Pahl et al., específicamente las fases de planificación y concepción, donde se establecieron los requisitos de diseño y el concepto del equipo para el tratamiento de efluentes por electrólisis. El equipo diseñado consistió en una cámara electrolítica con un raspador de espuma seguido de un decantador, además de los otros controles operativos. La alimentación y descarga se realizó mediante bombas centrífugas.

Palabras clave: Recurso hídrico; Tratamiento de aguas residuales; Tratamiento electrolítico; Impacto ambiental; Reutilizar.

\section{Introdução}

A água doce é um recurso essencial e um dos serviços ecossistêmicos mais valiosos (Lin et al., 2021). Segundo a Organização das Nações Unidas (ONU), estima-se que no mundo um bilhão de pessoas não tem acesso a um abastecimento suficiente de água por dia, ou seja, cerca de 20 litros por pessoa. A disponibilidade de água depende cada vez mais do gerenciamento e uso sustentável em todos os setores (Souza et al., 2020). A escassez, má distribuição e seu desperdício tem gerado grandes problemas socioeconômicos e isso faz com que o tema seja de preocupação global. A lavagem de carros é um processo que consome quantidade significativa de água e como a frota vem aumentando a cada ano, os impactos sobre os recursos hídricos devem ser considerados.

O reuso de água para a lavagem de carros vem ganhando destaque em vários países, pois verificou-se que milhares de litros de água potável são desperdiçados atualmente nesta prática. Alguns países como Estados Unidos e Japão tem uma legislação própria para a regulamentação de lavagens com tratamento de efluentes, utilizando equipamentos que promovam o reuso da água utilizada (Leitão, 1999). No Brasil, a obrigatoriedade de reuso de água na lavação de carros está crescendo, no entanto, ainda associada a algumas iniciativas isoladas.

O desenvolvimento de novas tecnologias é essencial para conseguir fazer um bom proveito e reuso deste recurso hídrico, contudo, o desafio para aumentar o reuso de água é o desenvolvimento de tecnologias eficientes, de fácil operação, sustentável e de baixo custo de implantação. Neste contexto, a eletrólise se apresenta como um processo promissor, mas carece de comprovação da sua aplicação em escala real.

Desta forma, o objetivo deste estudo é desenvolver um conceito de um equipamento para o tratamento de efluentes na lavagem de carros por eletrólise para posterior construção de um protótipo. A água depois de tratada será reutilizada para uma nova lavagem e a utilização de placas de energia solar também é um dos requisitos para este projeto, aumentando a sua sustentabilidade.

Para desenvolver este conceito utilizou-se a metodologia de desenvolvimento de produto proposta por Pahl et al., (2005), que propõe uma metodologia de projeto como um procedimento planejado e com indicações concretas de condutas a serem tomadas em seu desenvolvimento. Outro ponto importante mencionado pelo autor é o desenvolvimento de um produto no momento certo e que tenha mercado para ele.

\subsection{Impacto Ambiental}

Para Sánchez (2013) qualquer resultante causada por atividades humanas com alterações nas propriedades físicas, químicas ou biológicas, que afetam na qualidade dos recursos ambientais é considerada um impacto ambiental. Segundo Berté (2013), os impactos ambientais envolvem o homem e a natureza, são classificados como diretos ou indiretos, positivos ou negativos, ocasionais ou permanentes, locais ou globais e tem a poluição como sua consequência mais grave.

A água é fundamental para a sobrevivência de todas as formas de vida do planeta. Para o ser humano se manter vivo é necessário consumir alguns litros de água potável por dia. Porém, a água doce disponível no planeta não é abundante, pois ela representa apenas $0,01 \%$ da água total do planeta (Baird, 2002). 
A lavagem de veículos com água potável oferece um grande perigo ao meio ambiente, pois além de consumir cerca de 150 litros para a lavagem de um veículo popular, ela descarrega no meio ambiente um efluente com substâncias tóxicas capazes de gerar danos ambientais. É necessário o tratamento das águas utilizadas nas estações de lavagem para que esses resíduos não causem impactos ambientais. De acordo com Braga (2002), todos os tipos de águas residuais causam impactos estéticos, fisiológicos e ecológicos, através das alterações das características dos corpos hídricos receptores envolvidos.

Outro fator significativo neste problema é o aumento da frota de veículos no Brasil. Segundo DENATRAN (2020), o Brasil possui mais de 57,4 milhões de automóveis em circulação no território nacional. A frota de veículos vem aumentando a cada ano, sendo previsto um crescimento de 2,6\% de 2019 para 2020 (SINDIPEÇAS, 2019). As maiores concentrações de veículos estão nas regiões metropolitanas, mas os municípios menores também estão com uma presença crescente de carros. Mais da metade dos veículos brasileiros estão concentrados na região sudoeste, cerca de $54,2 \%$ da frota brasileira.

\subsection{Características dos efluentes lançados no meio ambiente pela lavagem de veículos e parâmetros utilizados para o} tratar o efluente

A lavagem de carros é um serviço com bastante demanda no meio urbano. Conforme Teixeira (2003), o efluente lançado no meio ambiente pela lavagem de veículos pode conter quantidades significativas de óleo e graxas, sólidos em suspensão, metais tóxicos, surfactantes e substâncias orgânicas. Os metais tóxicos nas águas residuais dos automóveis vêm geralmente das lonas de freio, com poeira e areia sendo levada e vestígios surfactante que dificilmente são degradados no meio ambiente (Tajuddin et al., 2020). O descarregamento de águas residuais da lavagem de carros no sistema de água, traz riscos à saúde humana e de outros organismos.

A característica do efluente produzido na lavagem de veículos de acordo com Bonh (2014), normalmente apresenta aspectos físico-químicos de acordo com a Tabela 1.

Tabela 1. Caracterização de efluente de lavagem de veículos.

\begin{tabular}{cccc}
\hline PARÂMETROS & \multicolumn{2}{c}{ RESULTADOS } & $\begin{array}{c}\text { RES. CONSEMA } \\
\text { 355/2017 }\end{array}$ \\
\hline & $\begin{array}{c}\text { Gonder et al., } \\
(\mathbf{2 0 1 7})\end{array}$ & Bohn (2014) & \\
\hline Detergentes -MBAS (mg/L) & 35,0 & 6,9 & 2,0 \\
\hline DQO $(\mathrm{mg} / \mathrm{L})$ & 560 & 354 & 330 \\
\hline Óleos e graxas $(\mathrm{mg} / \mathrm{L})$ & 125,0 & 19,4 & 10 \\
\hline Sólidos em suspensão -SST (mg/L) & 230,0 & 34,0 & 140 \\
\hline Materiais sedimentáveis $(\mathrm{mL} / \mathrm{L} / \mathrm{h})$ & - & 0,10 & 1 \\
\hline $\mathrm{pH}$ & 8,0 & 10,3 & 6 a 9 \\
\hline
\end{tabular}

Fonte: Adaptado de Bohn (2014) e Gonder et al (2017).

A Tabela 1 evidencia que o efluente de lavação de veículos não atende os padrões da legislação para lançamento e, portanto, necessita de tratamento adequado. As altas concentrações de detergentes e óleos graxas caracterizam o potencial poluidor deste tipo de efluente.

De acordo com Brown (2000), para reutilizar as águas residuais de lavagem de veículos é necessário fazer a separação de areia, óleos e graxas. Pode ser empregado processos de tratamentos adicionais para melhorar a qualidade da água recuperada. Alguns desses processos são: osmose inversa e nanofiltração, ultrafiltração, adsorção de carvão ativado por ultrafiltração, adsorção de carvão ativado por ultrafiltração, oxidação eletroquímica, tratamento biológico, floculação- 
sedimentação e floculação-flotação.

O investimento, o custo de operação e a manutenção de algumas destas alternativas são bastante caras e geralmente apresentam uma baixa eficiência. A flotação tem mostrado mais vantagens e com um potencial mais elevado perante os outros processos (Zaneti et al., 2011). O tratamento físico-químico com adição de coagulantes apresenta boa eficiência, no entanto a operação exige conhecimentos e treinamentos específicos. Neste contexto a eletrólise pode ser uma alternativa promissora (Gonder et al., 2017).

Mancuso e Santos (2003) dividem o reuso da água em duas categorias: potável e não potável. De acordo com a ANBT (1997), a água utilizada para a lavagem de veículos se classifica como classe 1 (não potável para usos urbanos). Os parâmetros exigidos pela norma são de acordo com a Tabela 2.

Tabela 2. Parâmetros para reuso da Classe 1 de acordo com norma ABNT 13969/97.

\begin{tabular}{ccc}
\hline Descrição & \multicolumn{2}{c}{ Quantidade/Unidade } \\
\hline Turbidez & inferior a 5 & $\mathrm{NTU}$ \\
\hline Coliforme fecal & inferior a 200 & $\mathrm{NMP} / 100 \mathrm{~mL}$ \\
\hline Sólidos dissolvidos & inferior a 200 & $\mathrm{mg} / \mathrm{L}$ \\
\hline $\mathrm{pH}$ & entre 6 e 8 & unidade de $\mathrm{pH}$ \\
\hline Cloro residual & entre 0,5 e 1,5 & $\mathrm{mg} / \mathrm{L}$ \\
\hline
\end{tabular}

Fonte: Associação Brasileira de Normas Técnicas (1997).

A comparação das características do efluente de lavação de veículos (Tabela 1) com os exigidos para reuso (Tabela 2) mostra que o tratamento deste tipo de efluente deve priorizar a remoção de turbidez.

\subsection{Processo de Eletrólise}

O processo de eletrólise ocorre quando uma corrente elétrica contínua atravessa dois elétrons, fazendo a dissociação da água em oxigênio e hidrogênio, utiliza-se um eletrólito assegure a condutividade, permitindo a realização do processo (Zeng et al., 2010). A Figura 1 mostra a representação esquemática do processo de eletrólise.

Figura 1. Processo eletrolítico.

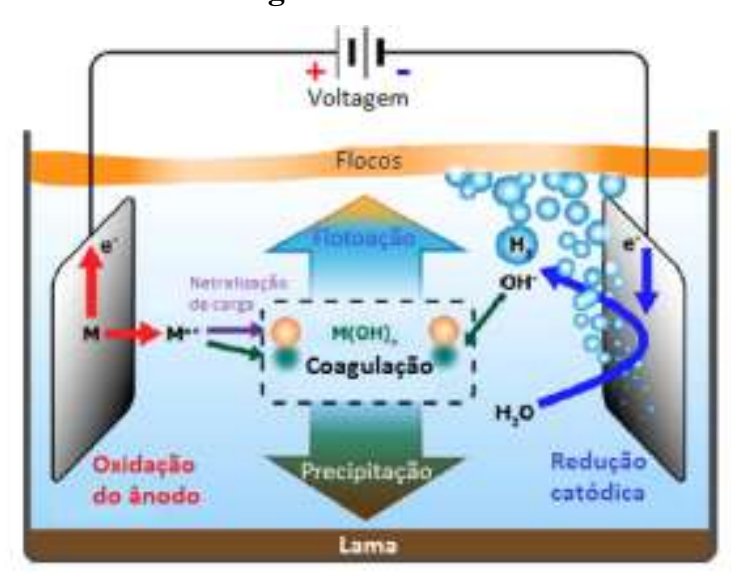

Fonte: Adaptado de AN, et al. (2016).

A passagem da corrente elétrica gera uma força eletromotriz capaz de provocar uma série de reações químicas, no caso característico para o tratamento de água, contribuem para a desestabilização das moléculas contaminantes, provocando a 
sua precipitação e/ou oxidação, mudando-as para compostos biodegradáveis (Bazrafshan et al, 2012).

Para o processo por meio de eletrólise existem diversas designações. Elas podem ser classificadas de acordo com o seu princípio:

a) Eletroflotação - De acordo com Chen (2003) e Leite (2007), a eletroflotação é um processo em que os poluentes flutuam para a superfície da água, através de minúsculas bolhas de gases hidrogênio e oxigênio ou cloro, gerados por eletrólise da água através de um reator eletroquímico.

b) Eletrofloculação - É a união dos processos de eletrocoagulação e eletroflotação. Segundo Mollah et al. (2001), o processo de eletrofloculção é altamente dependente da química do meio aquoso, especialmente pela condutividade.

Mollah et al. (2004) descreve as reações ocorridas num metal M resumidamente da seguinte maneira:

\section{No ânodo:}

$$
\begin{array}{lr}
\mathrm{M}_{(\mathrm{s})} \rightarrow \mathrm{M}_{(\mathrm{aq})} \mathrm{n}^{+}+\mathrm{ne}^{-} & \text {(Equação 1) } \\
2 \mathrm{H}_{2} \mathrm{O}_{(\mathrm{l})} \rightarrow 4 \mathrm{H}^{+}{ }_{(\mathrm{aq})}+\mathrm{O}_{2(\mathrm{~g})}+4 \mathrm{e}^{-} & \text {(Equação 2) }
\end{array}
$$

\section{No cátodo:}

$$
\begin{array}{ll}
\mathrm{M}_{(\mathrm{aq})} \mathrm{n}^{+}+\mathrm{ne}^{+} \rightarrow \mathrm{M}_{(\mathrm{s})} & \text { (Equação 3) } \\
2 \mathrm{H}_{2} \mathrm{O}_{(\mathrm{l})}+2 \mathrm{e}^{-} \rightarrow \mathrm{H}_{2(\mathrm{~g})}+2 \mathrm{OH}^{-} & \text {(Equação 4) }
\end{array}
$$

Ao se utilizares eletrodos de ferro ou de alumínio, os íons de $\mathrm{Fe}(\mathrm{aq}) 3+$ ou $\mathrm{Al}(\mathrm{aq}) 3+$ gerados submeter-se-ão imediatamente a reações espontâneas adicionais para produzir hidróxidos e/ou poli hidróxidos correspondentes, que realizam a coagulação dos contaminantes no efluente (MOLLAH et al., 2004; GONDER et al., 2017).

A densidade de corrente, o tempo e o tipo de eletrodo costumam ser as principais variáveis de estudo do processo eletrolítico aplicado ao tratamento de diferentes efluentes. Gonder et al., (2017) aplicou a eletrólise para tratar efluente de lavação de carros obtendo remoção de DQO e de Óleos e Graxas de 88\% e 68\%, respectivamente. Para esses resultados foram utilizados $\mathrm{pH}=6$, densidade de corrente de $1 \mathrm{~mA} / \mathrm{cm} 2$ e tempo de $30 \mathrm{~min}$.

\subsection{Desenvolvimento do conceito de projeto nas quatros etapas de Pahl \& Beitz}

O conceito do equipamento será desenvolvido através da metodologia de projeto segundo Pahl et al., (2005). O emprego de um método de projeto proporciona o planejamento, a análise de ferramentas e processos, métodos e configurações otimizadas de sistemas complexos. O produto a ser desenvolvido é um sistema compatível com a utilização de métodos de projeto e de desenvolvimento de produto, seu conceito será desenvolvido nas quatro etapas, abordando as fases de planejamento e concepção (Pahl et al., 2005). A Figura 2 ressalta as quatro fases do desenvolvimento de projeto conforme Pahl et al., (2005). 
Figura 2. Metodologia para desenvolvimento de projeto.

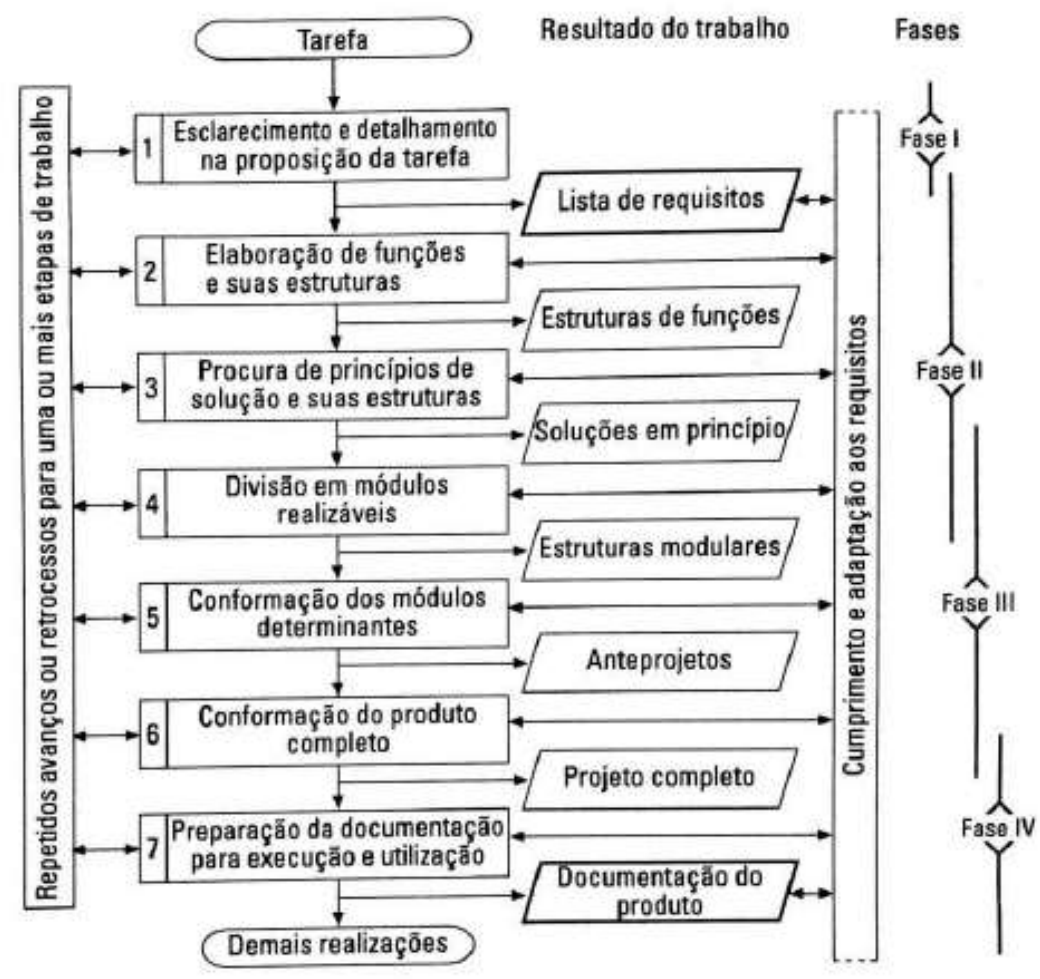

Fonte: Pahl et al., (2005).

\section{Metodologia}

Os resultados proporcionados nesta pesquisa são obtidos pela aplicação do método de elaboração de projeto elaborado por Pahl et al., (2005), na sequência a avaliação e a validação dos resultados obtidos com o desenvolvimento do projeto conceitual.

\subsection{Fase 1 Planejamento}

Inicialmente há a necessidade de explicar e detalhar as tarefas para dar o início projeto, para que não ocorra problemas e tenha que ser planejado novamente. Os requisitos de projeto serão descritos em uma lista de requisitos e observadas durante o seu desenvolvimento, onde defina-se ainda a sequência da tarefa desejada. Em primeiro lugar, tem-se como objetivo um equipamento que atenda a alguns requisitos mínimos como: reuso de água, fácil instalação, baixa manutenção e que atenda normas de vigentes de meio ambiente.

Avaliando as informações de como o equipamento deve funcionar, elabora-se uma lista que demostra os requisitos que o equipamento deve seguir. A lista de requisitos é elaborada a partir do método de Pahl et al., (2005) e não apresenta soluções e sim aspectos quantitativos e qualitativos.

O Quadro 1 demonstra os requisitos do projeto conforme a metodologia citada. Nele consta as características esperada do produto, sendo elas classificadas como desejáveis (D) ou exigíveis (E). 
Quadro 1. Lista de requisitos do projeto.

\begin{tabular}{|c|c|c|c|}
\hline Lista de req & tos pa & $\begin{array}{l}\text { o equipamento para o tratamento de efluentes de lavagem d } \\
\text { por eletrólise }\end{array}$ & \\
\hline DATA & E/D & EXIGÊNCIAS & \\
\hline & & 1-SISTEMA COM REÚSO DE ÁGUA & \\
\hline $03 / 12 / 2020$ & $\mathrm{E}$ & $\begin{array}{l}\text { Utilização de tanques para armazenamento da água da chuva para } \\
\text { reutilização na lavagem. }\end{array}$ & $\stackrel{\circ}{\circ}$ \\
\hline $03 / 02 / 2020$ & $\mathrm{E}$ & Reservatório com sistema de tratamento da água contaminada. & 完 \\
\hline & & 2-ENERGIA SUSTENTÁVEL & 桨 \\
\hline $30 / 06 / 2021$ & $\mathrm{D}$ & Utilização de placas solares para obtenção de energia fotovoltaica. & 됟 \\
\hline & & 3-INSTALAÇÃO & \\
\hline $03 / 12 / 2020$ & $\mathrm{E}$ & Equipamento adaptável em sistemas de lavagem & \\
\hline & & 4-SEGURANÇA & Z \\
\hline $03 / 12 / 2020$ & $\mathrm{E}$ & Atender as normas vigentes de segurança (NR $10,12, \ldots$ ) & \\
\hline & & 5-MEIO AMBIENTE & \\
\hline $03 / 12 / 2020$ & $\mathrm{E}$ & Atender as normas vigentes de meio ambiente & 留 \\
\hline & & 6-PRODUÇÃO & 䨔 \\
\hline $30 / 06 / 2020$ & E & Projeto simples e de baixo custo de produção & \\
\hline $30 / 06 / 2020$ & $\mathrm{D}$ & Produto modulável & \\
\hline & & 7-MANUTENÇÃO & \\
\hline $30 / 06 / 2020$ & D & Possibilitar remoção, substituição e reinstalação & \\
\hline
\end{tabular}

Fonte: Autores (2021).

A lista de requisitos foi definida a partir dos pontos que se espera do produto e ela apresenta uma série de exigências que são muito relevantes para o âmbito do projeto.

\subsection{Fase 2 Concepção}

A definição de concepção para Pahl et al., (2005) é a parte do projeto que após, o esclarecimento do problema, por isolamento dos problemas principais, a elaboração de estruturas da função e a busca de princípios de funcionamento apropriados, junto com a sua combinação na estrutura de funcionamento, define a solução preliminar da concepção e a definição de uma solução inicial.

A fase de projeto conceitual é composta das seguintes etapas:

1. Abstração para identificação dos problemas essenciais;

2. Elaboração da estrutura de funções, função global - Subfunções principais e secundárias;

3. Procura de princípio de trabalho para atendimento das subfunções;

4. $\quad$ Combinação dos princípios de trabalho para a estrutura de funções;

5. $\quad$ Seleção de combinações apropriadas;

6. Concretização de variantes de soluções de ideias;

7. Avaliação segundo critérios técnicos e econômicos;

8. Determinação de solução inicial.

Utilizando a lista de requisitos desenvolvido na fase 1 da metodologia de Pahl et al., (2005), defina-se a função global que o produto deve seguir. O fluxograma da Figura 3 apresenta as entradas, saídas, a função global encontrada e as subfunções 
necessárias para o atendimento da função principal. Como o problema de tratamento de efluente está bem definido, não foi realizado o procedimento da abstração.

Figura 3. Especificações da função global do produto e subfunções.

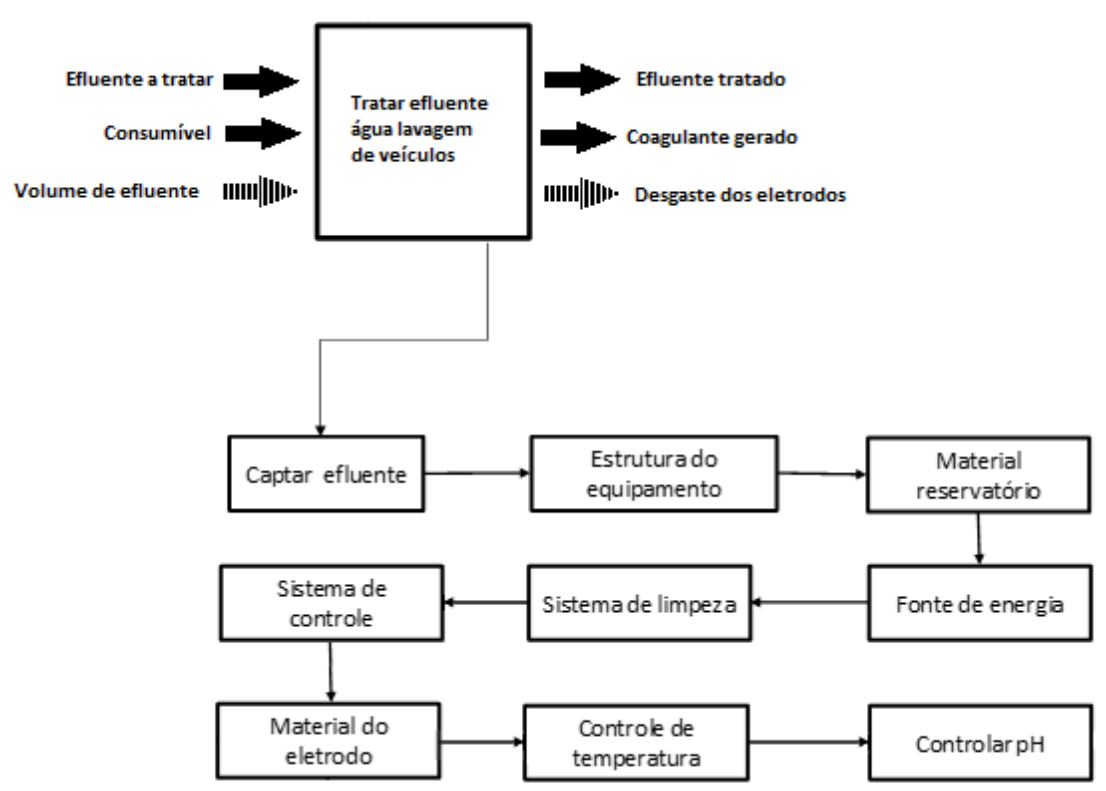

Fonte: Autores (2021).

\subsection{Variante da solução}

O quadro com as subfunções (Quadro 2) está montado com 9 linhas e 3 colunas de solução, algumas não possuem uma solução ideal, por este motivo não estão preenchidas. As soluções foram mostradas de uma forma clara, para obter um melhor entendimento.

Apresentamos de forma sucinta as soluções propostas para cada subfunção:

a) Sistema de captação de efluente: Três possibilidades foram pensadas para a captação, com bomba centrífuga, bomba submersa e bomba pneumática.

b) Estrutura do equipamento: Para a estrutura do equipamento foi levantado duas opções, estrutura em aço e estrutura em alumínio. A estrutura em aço precisa de um revestimento superficial para evitar a corrosão, já a em alumínio é mais leve, porém tem maior custo.

c) Material do reservatório: Três opções foram levantadas, reservatório em polipropileno, fibra e inox, sendo o reservatório de polipropileno possível de fabricação, o de fibra é uma alternativa via injeção e o de inox que seria soldado.

d) Fonte de energia: A fonte de energia que alimentará os eletrodos, pode ser gerada através de uma fonte chaveada que tem melhor custo/benefício ou uma fonte regulável que permite uma maior variação dos parâmetros.

e) Sistema de limpeza: Para fazer a remoção da camada floculada será utilizado um sistema mecânico semelhante ao de um limpador de vidro ou um sistema de deslizamento pneumático.

f) Sistema de controle: O controle poderá ser utilizado de maneira manual, com acionamento individuais e devidos sensores ou por sistema de CLP que poderá ser criado um supervisório de controle de equipamento.

g) Material dos eletrodos: Os eletrodos aplicados na eletrólise podem ser de aço ou alumínio tendo resultados semelhantes, o fator que deve ser avaliado é o custo de cada um.

h) Controle de temperatura: É um dos fatores que podem influenciar no processo, sendo assim, podemos conceber 
com ou sem controle.

i) Controle de pH: Análogo ao controle de temperatura, é um dos fatores que pode influenciar o processo ser concebido com ou sem controle.

Seguindo com a metodologia de Pahl et al., (2005), foram determinadas as soluções e para cada subfunção evidenciada no mínimo duas soluções. Com isso chegou-se a duas variantes a V1 e V2, sendo a variante V1 composta pelas seguintes características:

- Sistema para captação do efluente: Bomba centrífuga;

- $\quad$ Estrutura do componente: Aço;

- Material do reservatório: Fibra de vidro;

- $\quad$ Fonte de energia: Regulável;

- $\quad$ Sistema de limpeza: Mecânico;

- $\quad$ Sistema de controle CLP;

- Material dos eletrodos: Alumínio;

- $\quad$ Controle de temperatura: Sem controle de temperatura;

- $\quad$ Controle de $\mathrm{pH}$ do efluente: Sem controle de $\mathrm{pH}$ do efluente.

E a variante V2:

- Sistema para captação do efluente: Bomba centrífuga;

- $\quad$ Estrutura do componente: Alumínio;

- Material do reservatório: Aço inox;

- $\quad$ Fonte de energia: Regulável;

- $\quad$ Sistema de limpeza: Mecânico;

- $\quad$ Sistema de controle CLP;

- Material dos eletrodos: Alumínio;

- $\quad$ Controle de temperatura: Termopar;

- $\quad$ Controle de $\mathrm{pH}$ do efluente: Com controle de $\mathrm{pH}$ do efluente. 
Quadro 2. Concepções alternativas.

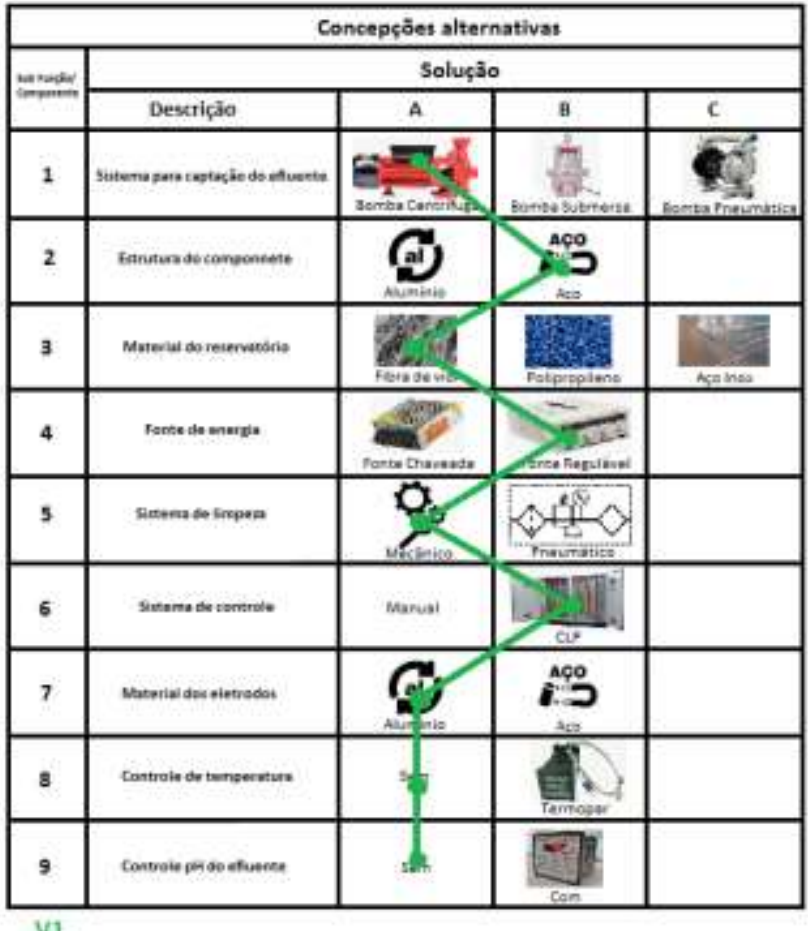

$V_{1}$

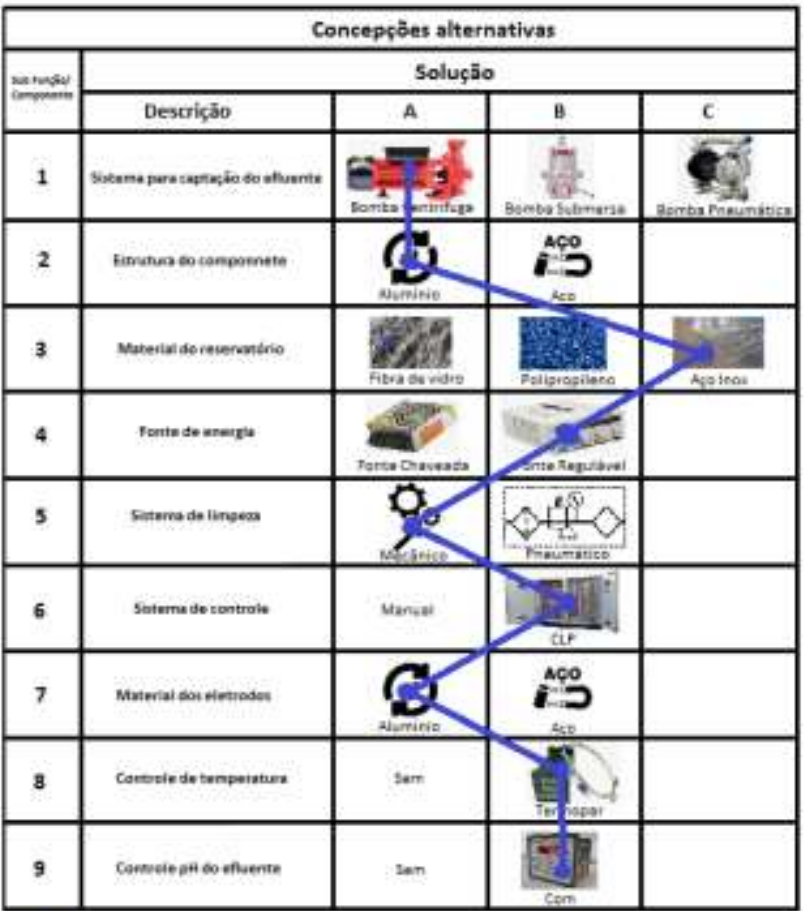

V2

Fonte: Autores (2021).

\section{Resultados e Discussão}

Por meio da lista de requisitos, desenvolvida na fase de planejamento de produto, juntamente com as escolhas de soluções na matriz ordenadora, houve a criação de duas variantes de solução, V1 e V2, onde as duas tem a mesma funcionabilidade, porém a V1 tem componentes mais básicos no seu projeto. O funcionamento das duas variantes consiste na captação da água utilizada na lavagem para reuso.

Conforme demonstrado na Figura 4, a água captada da lavagem será succionada por uma bomba centrífuga e destinada para um tanque onde terá um reator eletrolítico que fará o tratamento de efluente (Figura 5). O reator eletrolítico será composto por uma colmeia de eletrodos fabricados de alumínio e uma fonte com corrente contínua.

Após a passagem pelo reator eletrolítico, o efluente será armazenado no decantador. O efluente armazenado no decantador será novamente succionado por outra bomba centrífuga fazendo com que o efluente tratado retorne para o sistema de lavagem. O sistema de limpeza será composto por um motor de baixa potência, onde uma correia dentada ligada em uma lâmina de limpeza realizará a remoção da espuma gerada no processo de eletrólise. A vazão será controlada através de rotâmetros acoplados nos registros dos dois tanques de tratamento. O controle da pressão será monitorado por um manômetro. 
Figura 4. Concepção da variante V1.

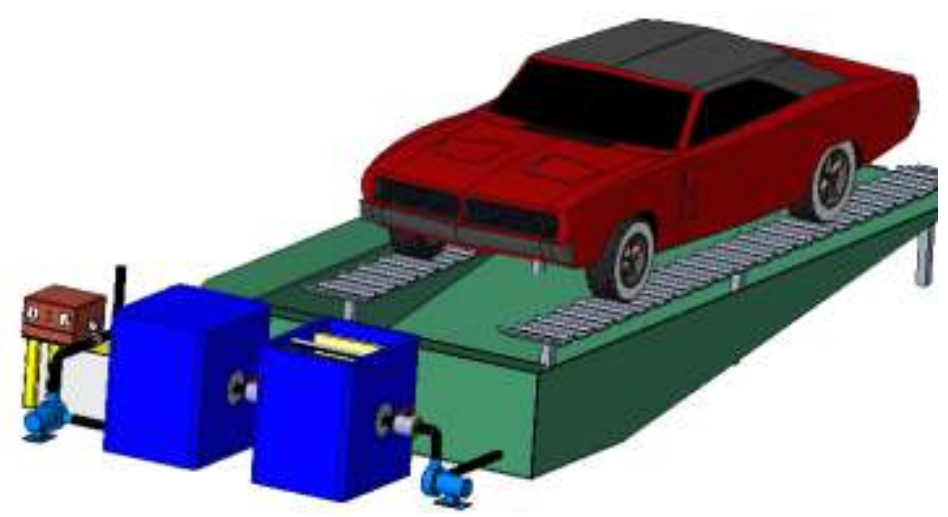

Fonte: Autores (2021).

Figura 5. Sistema de tratamento.

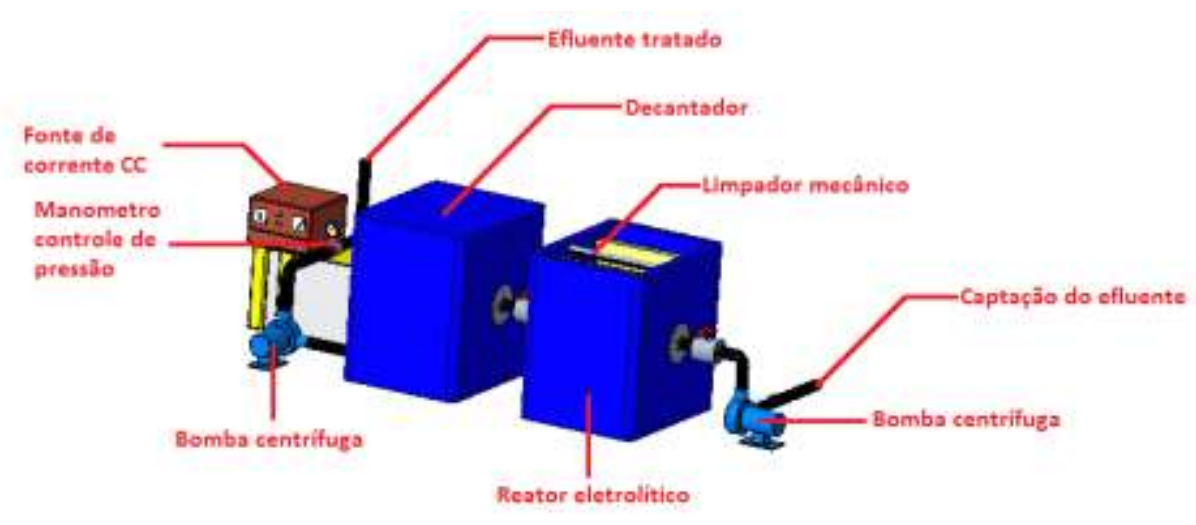

Fonte: Autores (2021).

As duas variantes são consideradas como sendo executáveis para o desenvolvimento do projeto e estas atenderiam a lista mestra. O projetista tem uma base bastante sólida para o sucesso do projeto e o desenvolvimento do produto. Adaptações de normas de segurança, seleção de materiais e custos são requisitos que serão realizados nas fases de projeto preliminar e detalhamento.

\section{Conclusão}

Um conceito de equipamento para o tratamento de efluente de lavagem de carros por eletrólise foi concebido a partir de soluções apresentadas através de uma matriz morfológica, sendo plausível estabelecer variantes de solução e seguir os requisitos escolhidos para o projeto. A utilização da metodologia, permitiu listar todas as características exigidas no projeto, mostrando clareza no seu desenvolvimento e o que se espera do produto, diminuindo bastante a necessidade de retrabalhos.

Com a aplicação das duas primeiras fases da metodologia de projeto, foi possível encontrar as melhores soluções para atender a lista de requisitos iniciais, possibilitando chegar a uma variante do produto segura e não por sentimento, capaz de direcionar a construção de um protótipo para aplicação em escala piloto em uma situação real, tendo como sugestão para trabalhos futuros a aplicação do protótipo em tamanho e situação real do dia a dia para assim comprovar o sucesso do projeto.

\section{Referências}

An, C., Huang, G., Yao, Y., \& Zhao, S. (2016). Emerging usage of electrocoagulation technology for oil removal from wastewater: A review. Science of the Total Environment, 579, 537-556. 
ABNT, Associação Brasileira de Normas Técnicas. (1997). NBR 13969: Tanques sépticos - Unidades de tratamento complementar e disposição final dos efluentes líquidos - Projeto, construção e operação. 60.

Baird, C. (2002). Química Ambiental. Boockman.

Bazrafshan, E., Moein, H., Mostafapour, F. K., Nakhaie, S. (2012). Application of Electrocoagulation Process for Dairy Wastewater Treatment. Journal of Chemistry, 2013, 1-8.

Braga, B. P. F., Hespanhol, I., Conejo, J. G. L., Barros, M. T. L., Junior, M. S. V., Porto, M. F. A., Nucci, N. L. R., Eiger, S., Juliano, N. M. A. (2002). Introdução à Engenharia Ambiental. São Paulo, 305.

Brown, C. (2000). Water Conservation in the Professional Car Wash Industry. https://www.carwash.org/docs/default-document-library

Berté, R. (2013). Gestão sócio ambiental no Brasil. Curitiba, PR: Intersaberes.

Bohn, F. P. (2014). Tratamento do Efluente Gerado na Lavagem de Veículos. Monografia (Graduação) - Universidade Regional do Noroeste do Estado do Rio Grande do Sul, Curso de Engenharia Mecânica, Panambi, 2014.

Chen, G. (2003). Electrochemical Technologies in Wastewater Treatment. Separation and Purification Technology, 38(1), 11-41.

Denatran. (2020). Frota nacional. http://www.denatran.gov.br/frota2020.htm.

Gonder, Z. B., Balioglu, G., Vergili, I., Kaya, Y. (2017). Electrochemical treatment of carwash wastewater using Fe and Al electrode: Techno-economic analysis and sludge characterization. Journal of Environmental Management, 200, 380-390.

Holt, P. (2002). Electrocoagulation: Unravelling and synthesising the mechanisms behind a water treatment process. Dissertação (Doutorado) - Universidade de Sidney, Departamento de Engenharia Química, Sidney, 2002.

Leitão, S. A. M. (1999). Bases para a estruturação das atividades de reúso de água no Brasil - Estágio atual, Artigo apresentado no II Encontro das Águas, Montevideu, Uruguai.

Leite, J. C. A., Vilar, E. O., Cavalcanti, E. B., Sales Filho, I. O. (2007). Aplicação da Eletroflotação para Remoção de Óleo Emulsionado em Águas de produção de Campo de Petróleo. $4^{\circ}$ PDPETRO, Campinas, São Paulo.

Lin, J., Huang, J., Prell, C., Bryan, B. A. (2021). Changes in supply and demand mediate the effects of land-use change on freshwater ecosystem services flows. Science of the Total Environment, 763, 143012.

Mancuso, P. C. S.; SANTOS, H. F (2003). Reuso de água. Barueri, SP: Manole.

Mollah, M. Y. A., Schennach, R., Parga, J. R., Cocke, D. L. (2001). Electrocoagulation (EC) - science and applications. Journal of Hazardous Materials, 84, $29-41$.

Mollah, M. Y. A., Morkovsky, P., Gomes, J. A. G., Kesmez, M., Parga, J., Cocke, D. L. (2004). Fundamentals, present and future perspectives of electrocoagulation. Journal of Hazardous Materials, 114(1-3), 199-210.

Pahl, G., Beitz, W., Feldhusem, J., Grote, K. H. (2005). Projeto na engenharia: fundamentos do desenvolvimento eficaz de produtos, métodos e aplicações. São Paulo, 1, 411 .

Resolução Consema/RS no 355/2017 (2017). Dispõe sobre os critérios e padrões de emissão de efluentes líquidos para as fontes geradoras que lancem seus efluentes em águas superficiais no Estado do Rio Grande do Sul. Porto Alegre, RS.

Sanchez, L. E. (2013). Avaliação de impacto ambiental: conceitos e métodos. Oficina de Textos.

Sindipeças (2019). https://www.sindipecas.org.br/sindinews/Economia/2019.

Souza, C. F., Hemkemeier, M. (2020). Pós tratamento por eletrocoagulação de efluente do processamento do soro de leite tratado por reator anaeróbio. Research, Society and Development, 9(9), e623997509.

Tajuddin, M. F., Al-Gheethi, A., Mohamed, R., Noman, E., Talip, B. A., Bakar, A. (2020). Optimizing of heavy metals removal from car wash wastewater by chitosan-ceramic beads using response surface methodology. Materials Today: Proceedings, 31, 43-47.

Teixeira, C. (2003). Emprego da filtração por ar dissolvido no tratamento de efluentes de lavagem de veículos visando a reciclagem a água. Dissertação (Mestrado). Universidade Estadual de Campinas, Faculdade de Engenharia Civil, Campinas, 2003.

Zaneti, R., Etchepare, R., Rubio, J. (2011). Car wash wastewater reclamation. Full-scale application and upcoming features. Resources, Conservation and Recycling, 55(11), 953-959.

Zeng, K., Zhang, D. (2010). Recent progress in alkaline water electrolysis for hydrogen production and applications. Progress in Energy and Combustion Science, 36(3), 307- 326. 